\title{
Neural correlates of attentional control in social anxiety disorder: the impact of early-life adversity and DNA methylation
}

\author{
Ariane Wiegand, MSc; Matthias H. J. Munk, MD; Sanja Drohm, MSc; \\ Andreas J. Fallgatter, MD; Julia L. MacIsaac, PhD; Michael S. Kobor, PhD; \\ Vanessa Nieratschker, PhD; Benjamin Kreifelts, MD
}

\begin{abstract}
Background: Social anxiety disorder is characterized by intense fear and avoidance of social interactions and scrutiny by others. Although alterations in attentional control seem to play a central role in the psychopathology of social anxiety disorder, the neural underpinnings in prefrontal brain regions have not yet been fully clarified. Methods: The present study used functional MRI in participants (age 18-50 yr) with social anxiety disorder ( $n=42,31$ female) and without ( $n=58,33$ female). It investigated the interrelation of the effects of social anxiety disorder and early-life adversity (a main environmental risk factor of social anxiety disorder) on brain activity during an attentional control task. We applied DNA methylation analysis to determine whether epigenetic modulation in the gene encoding the glucocorticoid receptor, NR3C1, might play a mediating role in this process. Results: We identified 2 brain regions in the left and medial prefrontal cortex that exhibited an interaction effect of social anxiety disorder and early-life adversity. In participants with low levels of early-life adversity, neural activity in response to disorder-related stimuli was increased in association with social anxiety disorder. In participants with high levels of early-life adversity, neural activity was increased only in participants without social anxiety disorder. NR3C1 DNA methylation partly mediated the effect of social anxiety disorder on brain activity as a function of early-life adversity. Limitations: The absence of behavioural correlates associated with social anxiety disorder limited functional interpretation of the results. Conclusion: These findings demonstrate that the neurobiological processes that underlie social anxiety disorder might be fundamentally different depending on experiences of early-life adversity. Long-lasting effects of early-life adversity might be encoded in NR3C1 DNA methylation and entail alterations in social anxiety disorder-related activity patterns in the neural network of attentional control.
\end{abstract}

\section{Introduction}

Social anxiety disorder is a psychiatric disorder characterized by excessive anxiety in social situations, centring around the fear of embarrassment and negative evaluation. A key element in the psychopathology of social anxiety disorder is a distortion in information processing that involves alterations of attentional control with an unintentional deployment of attentional resources toward disorder-related stimuli. ${ }^{1}$ In recent years, many studies have investigated the neural underpinnings of anxiety-related stimulus processing (e.g., emotional faces, ${ }^{2}$ self-referential comments ${ }^{3}$ or social anxiety disorder-related words ${ }^{4,5}$ ) in social anxiety disorder. Perception of such stimuli consistently involves hyperactivity in the amygdala and insular cortex, ${ }^{6}$ but also altered activation of the anterior cingulate, the parietal cortex, the hippocampus and the fusiform gyrus. ${ }^{7}$ Alterations in activity in the ventrolateral cortex, the dorsolateral pre- frontal cortex and the mediofrontal cortex have often been reported in association with reduced ability to engage in top-down attentional control in social anxiety. Unaccountably, however, findings in the frontal cortex were inconsistent because both decreased and increased hemodynamic activation were observed. ${ }^{7,8}$

A meta-analysis identified the inferior frontal junction in the lateral prefrontal cortex as one of the key regions of the attentional system. ${ }^{9}$ It also reported that the anterior insula, the anterior midcingulate cortex and presupplementary motor area were consistently associated with attentional control. Therefore, certain convergent brain regions have been found to be dysregulated in social anxiety disorder that generally play a key role in attentional control.

One of the most prevalent contextual factors of social anxiety disorder is early-life adversity. ${ }^{10}$ The early life is thought to be a particularly sensitive period, because the brain and its neural circuits are still developing. Disruptions by adverse

Correspondence to: A. Wiegand, Department of Psychiatry and Psychotherapy, Molecular Psychiatry, Tübingen Center for Mental Health, University Hospital Tübingen, Calwerstrasse 14, D-72076 Tübingen, Germany; ariane.wiegand@uni-tuebingen.de

Submitted Apr. 23, 2021; Revised Aug. 10, 2021; Accepted Oct. 10, 2021

Cite as: J Psychiatry Neurosci 2021 December 16;46(6). doi: 10.1503/jpn.210064 
events during this time window can have long-lasting behavioural and neurobiological effects. Behaviourally, alterations in attentional control ${ }^{11}$ and emotion processing ${ }^{11,12}$ have been reported; neuronally, early-life adversity has most consistently been associated with increased activity in the amygdala in response to threat-related stimuli, increased activation of the anterior cingulate cortex during emotion regulation, and altered lateral prefrontal activity in relation to executive functions. However, for the latter, the direction of effect varies among studies. ${ }^{13,14}$

Based on this strong congruence of brain regions that are dysregulated in association with both social anxiety disorder and early-life adversity - and on accumulating evidence that early-life adversity may represent an unrecognized confounding moderator in many psychiatric neuroimaging studies $^{14}$ — the goal of the present study was to investigate the interrelation of the effects of social anxiety disorder and early-life adversity on brain activity patterns.

The early life not only represents a period of increased neuroplasticity but also appears to be characterized by substantial developmental epigenetic plasticity. ${ }^{15}$ Epigenetic modifications present a mechanism for how environmental factors such as early-life adversity might affect gene regulation - for example, through DNA methylation. ${ }^{16}$ Primarily based on candidate gene studies motivated by work in rodents, the DNA methylation pattern of the NR3C1 gene has consistently been associated with both early-life adversity and anxiety disorders, including social anxiety disorder. ${ }^{17,18}$ NR3C1 encodes the glucocorticoid receptor, which plays a pivotal role in the body's stress response system along the hypothalamic-pituitary-adrenal axis. ${ }^{19}$ Although increased NR3C1 DNA methylation in association with early-life adversity has been observed in most studies, some have reported no associations or an opposite direction of effect. ${ }^{17}$

We investigated the interactions of social anxiety disorder and early-life adversity at the level of hemodynamic brain activation, focusing on attentional control. In particular, we examined whether the experience of early-life adversity might moderate activity differences associated with social anxiety disorder and, hence, might account for previously observed inconsistencies in brain activation patterns related to social anxiety disorder. We applied DNA methylation analysis to determine whether the DNA methylation of the NR3C1 gene might serve as a potential mediator of the effects of early-life adversity on brain function related to social anxiety disorder.

\section{Methods}

\section{Participants}

We screened 119 native German-speaking participants (age 18-50 yr) of European descent for study inclusion. All participants underwent the German version of the Structured Clinical Interview for DSM-IV. ${ }^{20}$ Of those, 48 participants had a primary diagnosis of social anxiety disorder. We evaluated the severity of participants' social anxiety using the Liebowitz Social Anxiety Scale. ${ }^{21}$ Six individuals with a diagnosis of social anxiety disorder but a low Liebowitz
Social Anxiety Scale score $\left(<35^{22}\right)$ and 13 without a diagnosis of social anxiety disorder but a high Liebowitz Social Anxiety Scale score $(\geq 35)$ were not included in our sample because of this inconsistency. We assessed early-life adversity using the Childhood Trauma Questionnaire, which measures 5 types of maltreatment occurring up to adolescence: emotional and physical neglect, and emotional, physical and sexual abuse. Participants who had at least a moderate score in 1 of the 5 categories (i.e., $\geq 15$ for emotional neglect, $\geq 13$ for emotional abuse, $\geq 10$ for physical abuse or neglect, $\geq 8$ for sexual abuse) were classified as having a high level of early-life adversity. ${ }^{23,24}$ Thus, 4 groups emerged: controls with low $(n=34)$ and high $(n=24)$ levels of early-life adversity, and participants with social anxiety disorder and low $(n=21)$ and high $(n=21)$ levels of early-life adversity. We assessed general anxiety using the State-Trait Anxiety Inventory ${ }^{25}$ and depressive symptoms using the Beck Depression Inventory. ${ }^{26}$ A sample description is detailed in Appendix 1, Table S1, available at jpn.ca.

All participants provided written informed consent before study inclusion. The study was performed in accordance with the Declaration of Helsinki in its latest version and approved by the University of Tübingen local ethics committee.

\section{Stimuli and task}

Participants conducted an emotional counting Stroop task. Stimuli comprised 60 words from 4 different categories (15 each): neutral, social anxiety disorder-related, fear-related and generally negative words. Each stimulus was presented in 3 conditions (i.e., repeated 2, 3 or 4 times), resulting in 180 stimulus events. Each stimulus was presented for $500 \mathrm{~ms}$. The mean intertrial interval was $6 \mathrm{~s}$ (range 4.5-7.5 s). For further details, see Appendix 1.

\section{Image acquisition}

We acquired MRI data using a 3 T scanner (PRISMA, Siemens). We recorded structural magnetization-prepared rapid gradient echo $T_{1}$-weighted images using the following parameters: 208 slices, repetition time $2400 \mathrm{~ms}$, echo time $2.22 \mathrm{~ms}$, voxel size $0.8 \times 0.8 \times 0.8 \mathrm{~mm}^{3}$. For functional imaging, we used multiband echo-planar imaging sequences with the following parameters: 72 slices, $2 \mathrm{~mm}$ thickness, repetition time $1500 \mathrm{~ms}$, echo time $34 \mathrm{~ms}$, voxel size $2 \times 2 \times 2 \mathrm{~mm}^{3}, 850$ volumes. For further details, see Appendix 1 .

\section{Experimental procedure}

Study participation included 2 appointments within 1 week. In the first session, participants were screened for current or previous psychiatric disorders using the Structured Clinical Interview for DSM-IV; ${ }^{20}$ completed several questionnaires related to basic demographic data, anxiety, depression and early-life adversity; and provided a blood sample. In the second session, eligible participants took part in the functional MRI experiment. 


\section{Behavioural and sample analysis}

We analyzed behavioural data using SPSS Statistics (version 26.0; IBM), and we created graphics using $\mathrm{R}$ (version 3.5.1). ${ }^{27}$ We analyzed the effects of social anxiety disorder, early-life adversity and their interaction on the characteristics of the sample using a univariate analysis of variance (ANOVA) with the dichotomous factors social anxiety disorder and early-life adversity, as well as their interaction. Because of technical problems, responses were not recorded for 6 participants. We excluded reaction times for incorrect trials, trials with multiple responses and trials with a reaction time more than 2 standard deviations from the mean reaction time that was within each word category (exclusion of about $6 \%$ of trials). We analyzed the mean reaction time for neutral and social anxiety disorder-related words in an ANOVA, with word category as the repeated-measures factor; early-life adversity and social anxiety disorder as between-subject factors; and age and sex as covariates. Similarly, we analyzed the number of correct trials with only 1 response given in an ANOVA, with word category as the repeated-measures factor; early-life adversity and social anxiety disorder as betweensubject factors; and age and sex as covariates. We performed post hoc $t$ tests to further investigate interaction effects.

\section{Functional MRI data analysis}

We preprocessed and analyzed the data with SPM12 (Wellcome Centre for Human Neuroimaging; www.fil.ion.ucl. ac.uk/spm/). Preprocessing comprised realignment, unwarping using a voxel displacement map derived from the field map, normalization to Montreal Neurological Institute space $^{28}$ and smoothing with a $6 \mathrm{~mm}$ full width at half maximum Gaussian function. At the single-participant level, we defined a general linear model with 1 regressor for each stimulus event and each realignment parameter. A high-pass filter removed frequencies below $1 / 128 \mathrm{~Hz}$. We estimated a first-order autoregressive model to account for serial correlations, and we calculated a contrast image for social anxiety disorder-related versus neutral stimuli. At the group level, we performed a full factorial ANOVA, with social anxiety disorder and early-life adversity as between-subject factors and age and sex as covariates. We applied a cluster-forming threshold of $p<0.001$ (uncorrected) and filtered it for clusters that consisted of 10 or more voxels.

We performed hypothesis-based small-volume correction using a predefined region of interest and assessed significance at a cluster level using a threshold of $p<0.05$ (family-wise error [FWE] corrected). ${ }^{29,30}$ This region of interest included brain regions that were activated in a metaanalysis of attentional control in the context of Stroop tasks (i.e., the inferior frontal junction in the lateral prefrontal cortex, the anterior insula, the anterior midcingulate cortex and the presupplementary motor area). ${ }^{9}$ We extracted mean contrast estimates from the significant clusters identified in the region of interest analysis and performed posthoc $t$ tests to further investigate interaction effects. To examine linear associations with the severity of early-life adversity, we assessed post hoc Pearson correlation to test for an association between the mean contrast estimates and the total and subscale scores on the Childhood Trauma Questionnaire (separately for participants with and without social anxiety disorder). Results from this correlation analysis underwent Bonferroni correction for multiple testing. We used Fisher $z$-transformation to test whether correlation coefficients differed between participants with and without social anxiety disorder.

\section{DNA methylation analysis}

DNA methylation analysis has been described in more detail in a previous report. ${ }^{31}$ Briefly, blood samples collected in EDTA tubes ( $9 \mathrm{~mL}$ Monovette; Sarstedt) were available from 84 participants (controls with low early-life adversity: $n=27$; controls with high early-life adversity: $n=18$; participants with social anxiety disorder and low early-life adversity: $n=$ 20; participants with social anxiety disorder and high earlylife adversity: $n=19$ ). DNA was extracted using the QIAamp Blood Maxi Kit (Qiagen) and bisulfite converted using the EZ DNA Methylation Kit (Zymo Research) according to the manufacturers' instructions. DNA methylation was assessed using the Infinium MethylationEPIC Kit (Illumina).

We performed data processing and analyses in $\mathrm{R}$ (version 3.5.1). ${ }^{27}$ We performed corrections for background and dye bias (Noob), ${ }^{32}$ probe design bias (BMIQ), ${ }^{33}$ batch effects (ComBat) $^{34}$ and cell type composition estimates (Houseman reference data set). ${ }^{35,36}$

DNA methylation data were filtered down to the $74 \mathrm{CpG}$ sites present on the EPIC array in the NR3C1 gene. For each site, we fitted a linear model, with early-life adversity and social anxiety disorder as main factors (as well as their interaction term) and age and sex as covariates (stats package). ${ }^{27}$ CpG sites with a $p$ value of less than 0.05 (false discovery rate corrected) for the interaction term were reported as significant and subjected to a conditional process analysis. We performed a moderated mediation analysis to investigate whether NR3C1 DNA methylation (continuous values) might mediate the effects of social anxiety disorder (dichotomous) on brain activity as a function of early-life adversity (dichotomous) using the SPSS implementation of PROCESS (model 8). ${ }^{37}$ PROCESS is a regression-based analysis tool for mediation, moderation and conditional process analyses. We used the latter application, estimating a conditional indirect effect in a moderated mediation model. We repeated functional MRI analyses in this subcohort as described in the previous section, and used mean contrast estimates from the significant cluster in the moderated mediation analysis as a continuous variable of brain activity.

\section{Results}

\section{Sample data}

Table 1 shows the sample characteristics for the 4 groups that emerged from the factors early-life adversity and social anxiety disorder, and the effects of social anxiety disorder, 


\begin{tabular}{|c|c|c|c|c|c|c|c|}
\hline \multirow[b]{2}{*}{ Characteristic } & \multicolumn{2}{|c|}{ Control* } & \multicolumn{2}{|c|}{ Social anxiety disorder ${ }^{\star}$} & \multirow[b]{2}{*}{$\begin{array}{l}\text { Effect of social } \\
\text { anxiety disorder }\end{array}$} & \multirow[b]{2}{*}{$\begin{array}{l}\text { Effect of early- } \\
\text { life adversity }\end{array}$} & \multirow{2}{*}{$\begin{array}{c}\text { Effect of } \\
\text { social anxiety } \\
\text { disorder } \times \text { early- } \\
\text { life adversity }\end{array}$} \\
\hline & $\begin{array}{l}\text { Low early-life } \\
\text { adversity } \\
n=34\end{array}$ & $\begin{array}{l}\text { High early-life } \\
\text { adversity } \\
n=24\end{array}$ & $\begin{array}{l}\text { Low early-life } \\
\text { adversity } \\
n=21\end{array}$ & $\begin{array}{l}\text { High early-life } \\
\text { adversity } \\
n=21\end{array}$ & & & \\
\hline Age, yr & $25.1 \pm 3.9$ & $27.6 \pm 6.7$ & $23.0 \pm 3.9$ & $26.3 \pm 8.2$ & $\begin{array}{c}F_{1,96}=2.08 \\
p=0.15\end{array}$ & $\begin{array}{c}F_{1,96}=6.39 \\
p=0.013\end{array}$ & $\begin{array}{c}F_{1,96}=0.12 \\
p=0.73\end{array}$ \\
\hline Sex, $n$ & $23 \mathrm{~F}, 11 \mathrm{M}$ & $10 \mathrm{~F}, 14 \mathrm{M}$ & $18 \mathrm{~F}, 3 \mathrm{M}$ & $13 \mathrm{~F}, 8 \mathrm{M}$ & $\begin{array}{c}F_{1,96}=4.07 \\
p=0.046\end{array}$ & $\begin{array}{c}F_{1,96}=6.88 \\
p=0.010\end{array}$ & $\begin{array}{c}F_{1,96}=0.01 \\
p=0.91\end{array}$ \\
\hline Education, yr & $17.72 \pm 3.23$ & $17.08 \pm 2.56$ & $15.42 \pm 4.56$ & $16.67 \pm 4.74$ & $\begin{array}{c}F_{1,88}=3.90 \\
p=0.05\end{array}$ & $\begin{array}{c}F_{1,88}=0.39 \\
p=0.53\end{array}$ & $\begin{array}{c}F_{1,88}=0.003 \\
p=0.96\end{array}$ \\
\hline $\begin{array}{l}\text { Liebowitz Social } \\
\text { Anxiety Scale score }\end{array}$ & $9.5 \pm 7.7$ & $11.3 \pm 8.3$ & $73.7 \pm 22.3$ & $79.4 \pm 21.6$ & $\begin{aligned} F_{1,96} & =443.14 \\
p & <0.001\end{aligned}$ & $\begin{array}{c}F_{1,96}=1.45 \\
p=0.23\end{array}$ & $\begin{array}{c}F_{1,96}=0.40 \\
p=0.53\end{array}$ \\
\hline $\begin{array}{l}\text { Childhood Trauma } \\
\text { Questionnaire score }\end{array}$ & $35.1 \pm 6.2$ & $50.5 \pm 13.3$ & $38.1 \pm 7.0$ & $64.0 \pm 18.4$ & $\begin{array}{c}F_{1,96}=11.88 \\
p<0.001\end{array}$ & $\begin{array}{c}F_{1,96}=74.47 \\
p<0.001\end{array}$ & $\begin{array}{c}F_{1,96}=4.89 \\
p=0.029\end{array}$ \\
\hline $\begin{array}{l}\text { State-Trait Anxiety } \\
\text { Inventory, state score }\end{array}$ & $31.12 \pm 5.59$ & $30.88 \pm 5.76$ & $45.33 \pm 12.75$ & $47.19 \pm 11.84$ & $\begin{array}{c}F_{1,96}=68.54 \\
p<0.001\end{array}$ & $\begin{array}{c}F_{1,96}=0.19 \\
p=0.66\end{array}$ & $\begin{array}{c}F_{1,96}=0.32 \\
p=0.57\end{array}$ \\
\hline $\begin{array}{l}\text { State-Trait Anxiety } \\
\text { Inventory, trait score }\end{array}$ & $30.03 \pm 6.46$ & $32.21 \pm 9.16$ & $52.52 \pm 12.18$ & $54.52 \pm 8.50$ & $\begin{array}{c}F_{1,96}=50.18 \\
p<0.001\end{array}$ & $\begin{array}{c}F_{1,96}=1.31 \\
p=0.26\end{array}$ & $\begin{array}{c}F_{1,96}=0.002 \\
p=0.96\end{array}$ \\
\hline $\begin{array}{l}\text { Beck Depression } \\
\text { Inventory II score }\end{array}$ & $1.44 \pm 2.38$ & $1.96 \pm 3.51$ & $13.57 \pm 10.58$ & $15.71 \pm 7.84$ & $\begin{array}{c}F_{1,96}=98.25 \\
p<0.001\end{array}$ & $\begin{array}{c}F_{1,96}=1.04 \\
p=0.31\end{array}$ & $\begin{array}{c}F_{1,96}=0.39 \\
p=0.54\end{array}$ \\
\hline IQ (MWT-B) & $105.15 \pm 13.54$ & $110.29 \pm 12.02$ & $109.33 \pm 13.69$ & $109.52 \pm 2.22$ & $\begin{array}{c}F_{1,96}=0.42 \\
p=0.52\end{array}$ & $\begin{array}{c}F_{1,96}=1.02 \\
p=0.32\end{array}$ & $\begin{array}{c}F_{1,96}=0.88 \\
p=0.35\end{array}$ \\
\hline
\end{tabular}

$\mathrm{F}=$ female; $\mathrm{M}=$ male; MWT-B = Mehrfachwahl-Wortschatz-Intelligenztest.
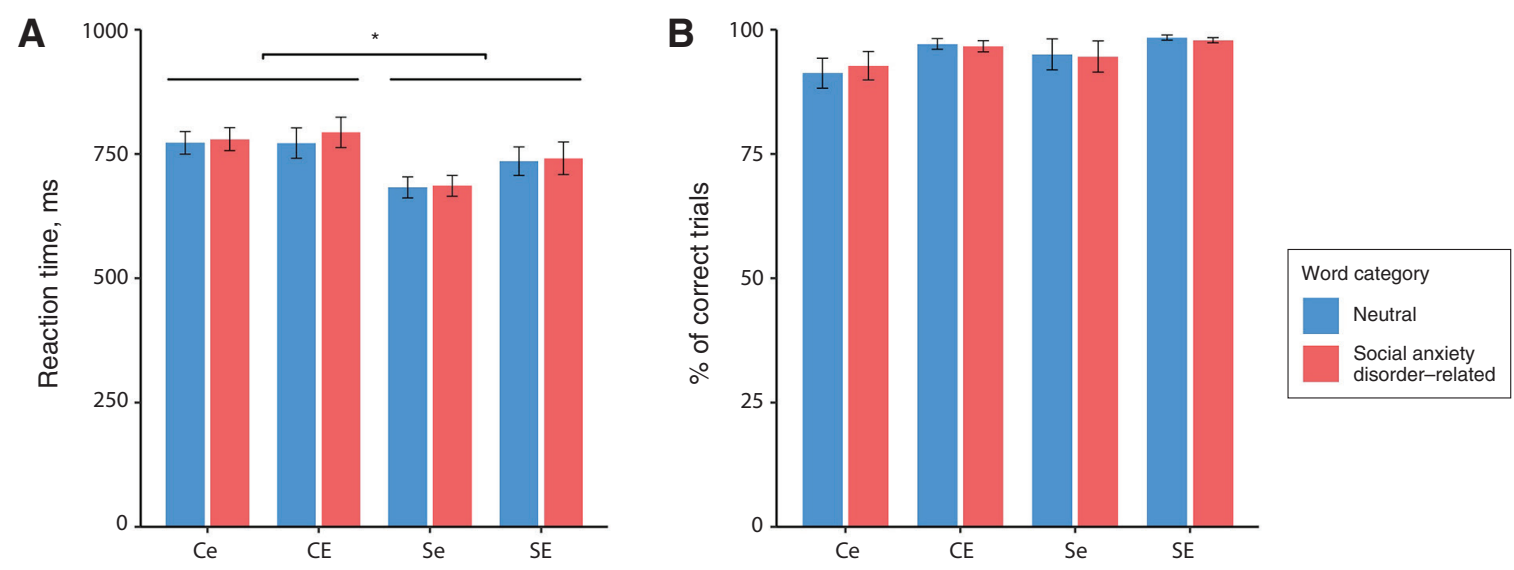

Figure 1: Emotional interference. (A) Mean reaction time ( \pm standard error) for each group. (B) Mean \% of correctly answered trials $( \pm$ standard error) for each group. $\mathrm{Ce}=$ control participants with low levels of early-life adversity; $\mathrm{CE}=\mathrm{control}$ participants with high levels of early-life adversity; $\mathrm{Se}=$ participants with social anxiety disorder and low levels of early-life adversity; SE = participants with social anxiety disorder and high levels of early-life adversity. ${ }^{*} p<0.05$.

early-life adversity and their interaction. We found a significant correlation between total score on the Liebowitz Social Anxiety Scale and total score on the Childhood Trauma Questionnaire $(r=0.35, p<0.001)$.

\section{Emotional interference}

Investigating reaction times for social anxiety disorder-related and neutral words, we found a significant main effect of social anxiety disorder $\left(F_{1,88}=6.60, p=0.012, \eta_{\mathrm{p}}{ }^{2}=0.070\right)$ but not of early-life adversity $\left(F_{1,88}=0.68, p=0.41\right)$. Further, we found no main effect of word category $\left(F_{1,88}=1.30, p=0.26\right)$ and no interaction between word category and social anxiety disorder $\left(F_{1,88}=3.88, p=0.052\right)$, word category and early-life adversity $\left(F_{1,88}=2.60, p=0.11\right)$ and no significant 3-way interaction $\left(F_{1,88}=0.13, p=0.72\right)$. Post hoc $t$ tests showed that participants with social anxiety disorder had significantly shorter reaction times than those without social anxiety disorder $\left(t_{92}>2.44, p<0.017\right)$. Figure $1 \mathrm{~A}$ shows the reaction times for the 2 word categories for each group. 
Table 2: Neural correlates associated with the interaction of social anxiety disorder and early-life adversity*

\begin{tabular}{lcccc}
\hline & MNI coordinates, & & Cluster size, \\
Structure & $x, y, z$ & $F_{1,94}$ & voxels & $p_{\text {FWE }}$ \\
\hline Left middle frontal gyrus & $-40,10,34$ & 20.07 & 68 & 0.007 \\
Left medial superior frontal gyrus & $-4,26,44$ & 18.75 & 36 & 0.036 \\
\hline
\end{tabular}

$\mathrm{FWE}=$ family-wise error; $\mathrm{MNI}=$ Montreal Neurological Institute.

* Significant clusters of differential activation within the a priori region of interest are described by anatomic structure, MNI coordinates and the $F$ value of the peak voxel. Voxel size: $2 \times 2 \times 2 \mathrm{~mm}^{3}$.

For the number of correct trials, we found no main effect of social anxiety disorder $\left(F_{1,88}=0.62, p=0.43\right)$ or early-life adversity $\left(F_{1,88}=0.02, p=0.90\right)$. We also found no main effect for word category $\left(F_{1,88}=0.75, p=0.39\right)$, and no significant interactions between word category and social anxiety disorder $\left(F_{1,88.19}=0.97, p=0.33\right)$, word category and early-life adversity $\left(F_{1,88}=2.72, p=0.10\right)$ and no significant 3 -way interaction $\left(F_{1,88}=1.56, p=0.22\right)$. Figure $1 \mathrm{~B}$ shows the number of correct trials for the 2 word categories for each group. See Appendix 1, Figure S1, for behavioural data for all 4 word categories.

\section{Functional MRI data}

We identified 2 clusters with a significant interaction between social anxiety disorder and early-life adversity in the left and medial prefrontal cortex for the contrast of social anxiety disorder-related and neutral stimuli (Table 2 and Figure 2). The third cluster indicated in Figure 2A (i.e., emerging at an uncorrected threshold of $p<0.001$ ) also partly overlapped with our predefined region of interest but was not significant in our region of interest analysis $\left(p_{\mathrm{FWE}}=0.063\right)$. We identified no significant associations with the main effects of social anxiety disorder and early-life adversity. At a descriptive level, we observed no clusters that surpassed the cluster-size threshold of 10 voxels or more outside the a priori region of interest.

The cluster in the left middle frontal gyrus extended to the inferior frontal and precentral gyrus, spanning the border of the ventrolateral and dorsolateral prefrontal cortex and the premotor cortex (Figure 2). Decomposition of the interaction effect in this cluster showed an increased activation difference between social anxiety disorder-related and neutral stimuli in association with social anxiety disorder in participants with low levels of early-life adversity $\left(t_{53}=\right.$ $-3.06, p=0.004)$; in contrast, we found decreased activation differences in association with social anxiety disorder in participants with high levels of early-life adversity $\left(t_{43}=\right.$ $3.47, p=0.001$; Figure 2). Exploratory analyses for the other word categories revealed similar trends. The contrasts of "fear-related words versus neutral words" and "negative words versus neutral words" showed no significant effect in participants with low levels of early-life adversity $\left(t_{53}=\right.$ $-0.75, p=0.46 ; t_{53}=-0.83, p=0.41$ ), but decreased activation differences in association with social anxiety disorder in participants with high levels of early-life adversity $\left(t_{43}=\right.$ $3.45, p=0.001 ; t_{38.7}=3.54, p=0.001$ ).
Decomposition of the interaction effect in the medial superior frontal gyrus cluster in the anterior part of the presupplementary motor area extending into the midcingulate cortex showed a similar pattern (Figure 2). For the contrast of "social anxiety disorder-related words versus neutral words," we found an increased activation difference in association with social anxiety disorder in participants with low levels of early-life adversity $\left(t_{53}=-2.98, p=0.004\right)$, and a decreased activation difference in association with social anxiety disorder in participants with high levels of early-life adversity $\left(t_{43}=3.32, p=0.002\right)$. Likewise, for the contrasts of "fear-related words versus neutral words" and "negative words versus neutral words," activation differences were increased with respect to social anxiety disorder in participants with low levels of early-life adversity $\left(t_{53}=-2.51, p=0.015\right.$; $t_{53}=-2.37, p=0.021$ ) and decreased (at least by trend) in participants with high levels of early-life adversity $\left(t_{43}=2.47, p=\right.$ $0.018 ; t_{40}=2.00, p=0.051$; Appendix 1, Figure S2).

Further validation analyses excluding confounds through depressive symptoms, general anxiety or alcohol abuse are reported in Appendix 1.

From a different perspective, Table 3 and Appendix 1, Figure S3, show the correlation of the experienced levels of early-life adversity (total and subcategories) with observed activation differences in the 2 identified clusters. Correlations of emotional neglect with the activity contrasts in both clusters differed significantly between participants with and without social anxiety disorder. Furthermore, correlations of emotional abuse and total score on the Childhood Trauma Questionnaire score with activity contrasts in the left middle frontal gyrus differed significantly between groups.

\section{DNA methylation differences and mediation analysis}

We investigated DNA methylation at the $74 \mathrm{CpG}$ sites in the NR3C1 gene (Appendix 1, Table S2). DNA methylation at $1 \mathrm{CpG}$ site located in intron 2 of the NR3C1 gene (specifically cg25708981) was associated with the interaction of social anxiety disorder and early-life adversity $\left(p_{\mathrm{FDR}}=0.037\right)$. In participants with high levels of early-life adversity, social anxiety disorder was associated with increased methylation levels $\left(t_{35}=-3.15, p=0.003\right.$; mean \pm standard deviation [SD]: controls with high levels of early-life adversity $91.87 \pm 1.47 \%$; participants with social anxiety disorder and high levels of early-life adversity $93.34 \% \pm 1.36 \%$ ). We found no significant difference in DNA methylation associated with social anxiety disorder in participants with low levels of early-life adversity 
A

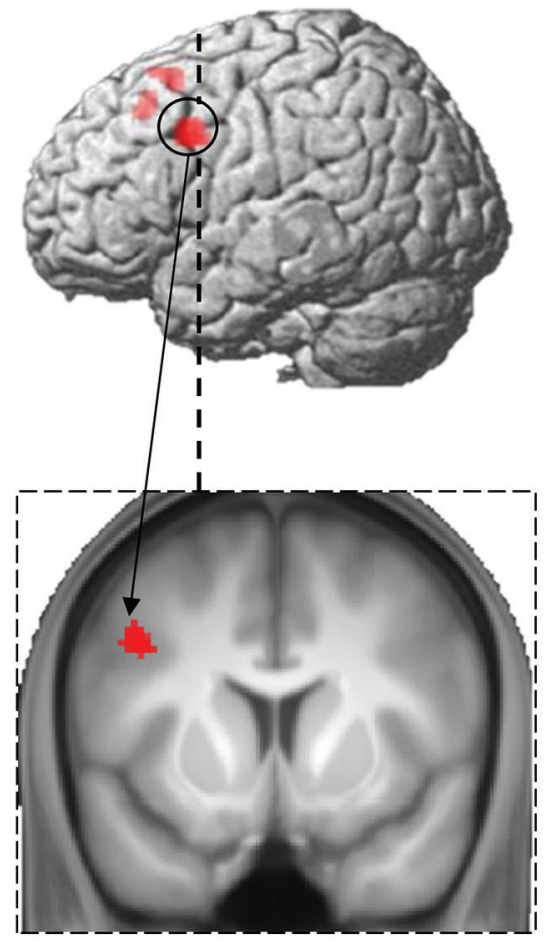

D

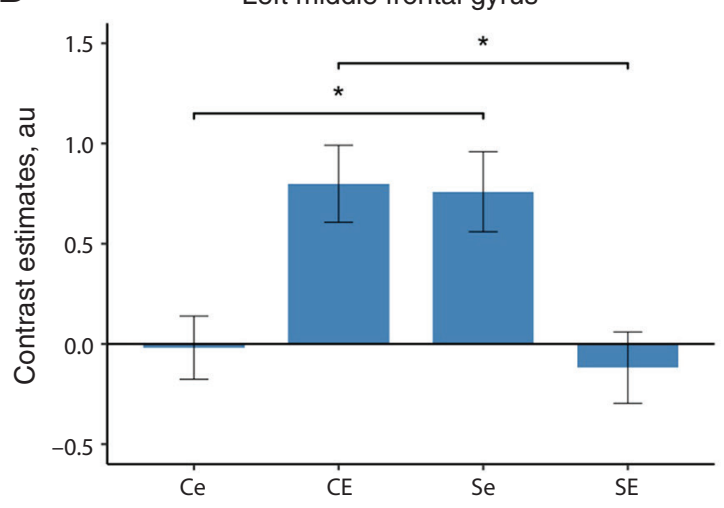

C

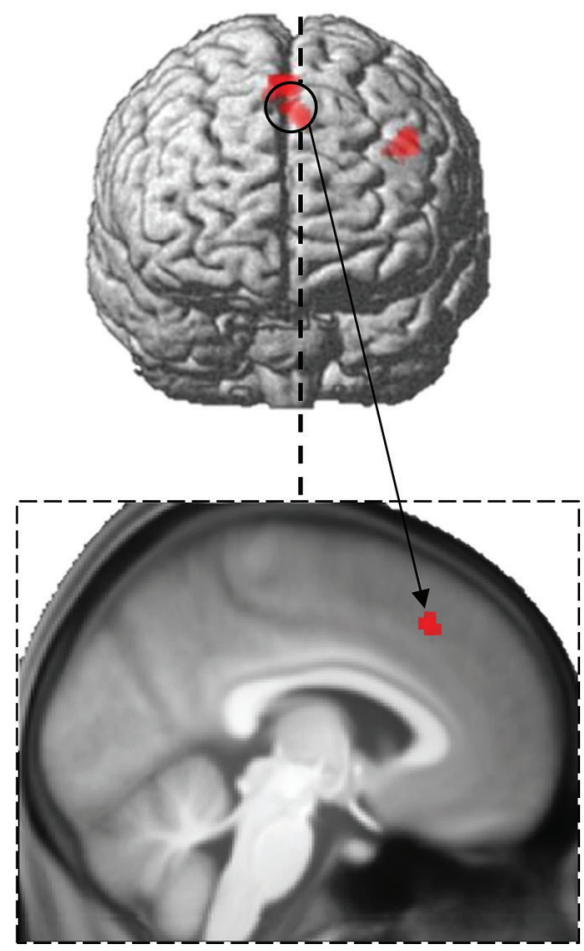

E Left medial superior frontal gyrus

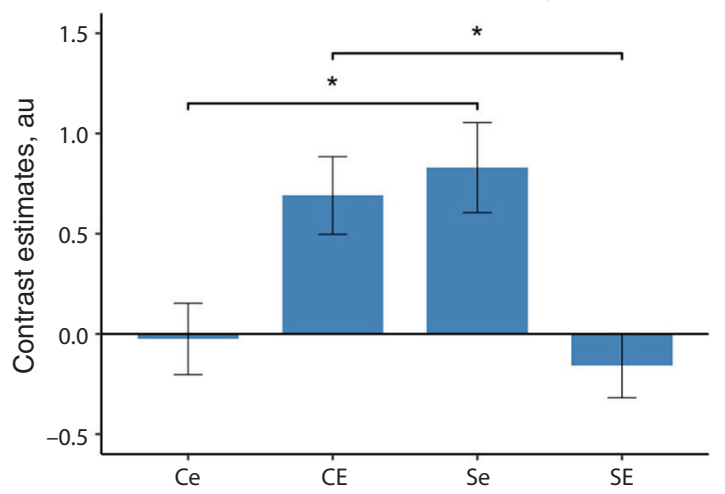

Figure 2: Neural correlates of the contrast "social anxiety-related words versus neutral words" associated with the interaction of social anxiety disorder and early life adversity. Clusters of differential activation at an uncorrected threshold of $p<0.001$ and filtered for clusters of 10 voxels or more, rendered on (A) a standard brain and (B) coronal and (C) sagittal slices of the whole sample mean anatomy. (D, E) Mean contrast estimates for each group extracted from the significant clusters identified in the region of interest analysis. Error bars depict standard errors of the mean; ${ }^{*} p<0.05$. $\mathrm{Ce}=$ control participants with low levels of early-life adversity; $\mathrm{CE}=$ control participants with high levels of early-life adversity; Se = participants with social anxiety disorder and low levels of early-life adversity; SE = participants with social anxiety disorder and high levels of early-life adversity.

$\left(t_{45}=1.97, p=0.055\right.$; controls $92.47 \pm 1.51 \%$; social anxiety disorder $91.58 \% \pm 1.57 \%$; Figure 3A). DNA methylation levels at this CpG site were comparable to a previous data set, where the mean $( \pm \mathrm{SD})$ DNA methylation was $91 \pm 6 \% .^{38}$
Investigation of brain activity patterns associated with the interaction of early-life adversity and social anxiety disorder in this subcohort (where DNA methylation data were available) identified 1 significant cluster in the left middle 


\begin{tabular}{|c|c|c|c|c|c|c|}
\hline \multirow[b]{2}{*}{$\begin{array}{l}\text { Childhood Trauma } \\
\text { Questionnaire scale }\end{array}$} & \multicolumn{3}{|c|}{ Left middle frontal gyrus } & \multicolumn{3}{|c|}{ Left medial superior frontal gyrus } \\
\hline & $\begin{array}{l}\text { Without social } \\
\text { anxiety disorder }\end{array}$ & $\begin{array}{l}\text { With social } \\
\text { anxiety disorder }\end{array}$ & $z / p$ & $\begin{array}{l}\text { Without social } \\
\text { anxiety disorder }\end{array}$ & $\begin{array}{l}\text { With social } \\
\text { anxiety disorder }\end{array}$ & $z / p$ \\
\hline Emotional abuse & $\begin{array}{c}r=0.43 \\
p=0.001^{\star}\end{array}$ & $\begin{array}{l}r=-0.19 \\
p=0.24\end{array}$ & $\begin{array}{c}z=3.12 \\
p=0.001^{\star}\end{array}$ & $\begin{array}{l}r=0.21 \\
p=0.12\end{array}$ & $\begin{array}{l}r=-0.20 \\
p=0.20\end{array}$ & $\begin{array}{c}z=1.99 \\
p=0.023\end{array}$ \\
\hline Physical abuse & $\begin{array}{l}r=0.25 \\
p=0.06\end{array}$ & $\begin{array}{l}r=-0.13 \\
p=0.41\end{array}$ & $\begin{array}{c}z=1.85 \\
p=0.033\end{array}$ & $\begin{array}{l}r=0.19 \\
p=0.16\end{array}$ & $\begin{array}{l}r=-0.11 \\
p=0.48\end{array}$ & $\begin{array}{l}z=1.45 \\
p=0.07\end{array}$ \\
\hline Sexual abuse & $\begin{array}{l}r=0.19 \\
p=0.15\end{array}$ & $\begin{array}{l}r=-0.20 \\
p=0.20\end{array}$ & $\begin{array}{c}z=1.89 \\
p=0.030\end{array}$ & $\begin{array}{l}r=0.14 \\
p=0.28\end{array}$ & $\begin{array}{l}r=-0.03 \\
p=0.85\end{array}$ & $\begin{array}{l}z=0.82 \\
p=0.21\end{array}$ \\
\hline Emotional neglect & $\begin{aligned} r & =0.30 \\
p & =0.022\end{aligned}$ & $\begin{array}{l}r=-0.31 \\
p=0.045\end{array}$ & $\begin{array}{c}z=3.01 \\
p=0.001^{*}\end{array}$ & $\begin{array}{c}r=0.29 \\
p=0.028\end{array}$ & $\begin{aligned} r & =-0.49 \\
p & =0.001^{*}\end{aligned}$ & $\begin{array}{c}z=3.99 \\
p<0.001^{*}\end{array}$ \\
\hline Physical neglect & $\begin{array}{l}r=0.22 \\
p=0.09\end{array}$ & $\begin{array}{l}r=-0.09 \\
p=0.55\end{array}$ & $\begin{array}{l}z=1.50 \\
p=0.07\end{array}$ & $\begin{array}{l}r=0.13 \\
p=0.34\end{array}$ & $\begin{array}{l}r=-0.30 \\
p=0.05\end{array}$ & $\begin{array}{c}z=2.10 \\
p=0.018\end{array}$ \\
\hline Total score & $\begin{array}{c}r=0.41 \\
p=0.001^{*}\end{array}$ & $\begin{array}{l}r=-0.30 \\
p=0.05\end{array}$ & $\begin{array}{c}z=3.56 \\
p<0.001^{*}\end{array}$ & $\begin{array}{l}r=0.19 \\
p=0.15\end{array}$ & $\begin{array}{l}r=-0.29 \\
p=0.07\end{array}$ & $\begin{array}{c}z=2.35 \\
p=0.010\end{array}$ \\
\hline
\end{tabular}

*Significant results (after correction for multiple testing). Fisher $\mathrm{z}$ transformation to compare correlations.

A

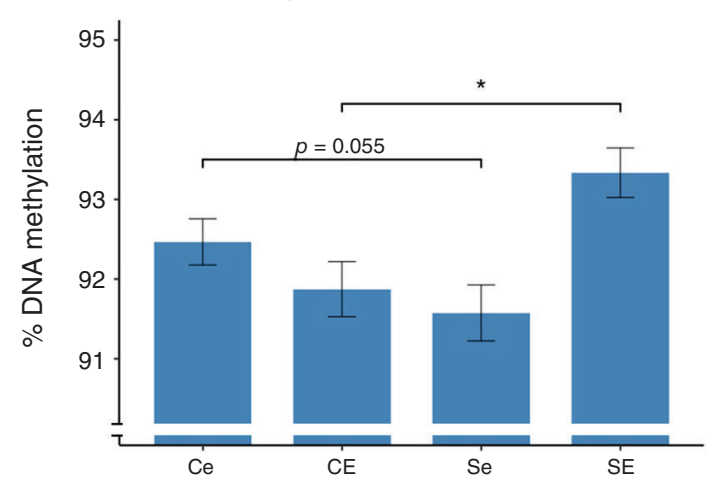

C

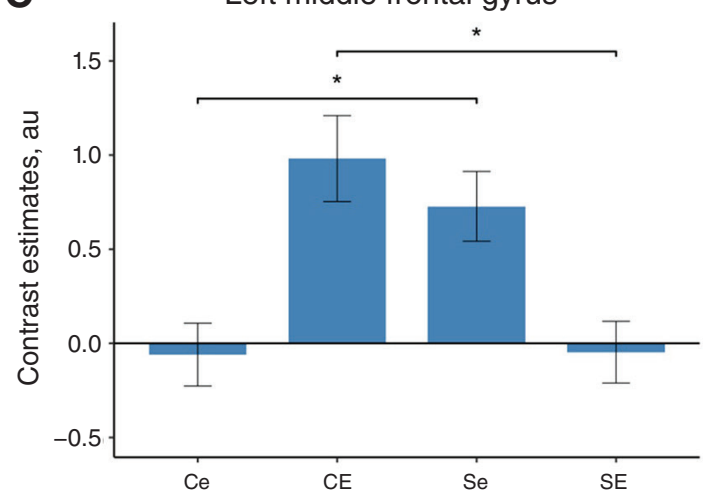

B

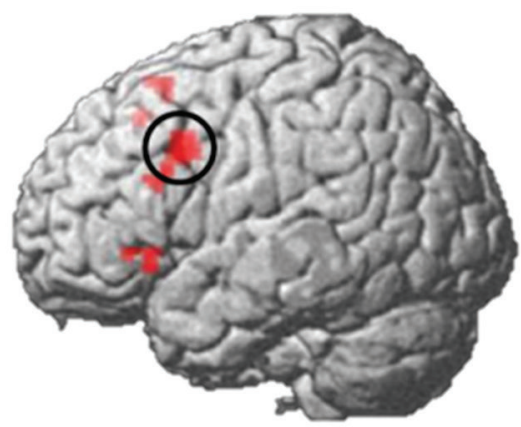

D

Moderated mediation

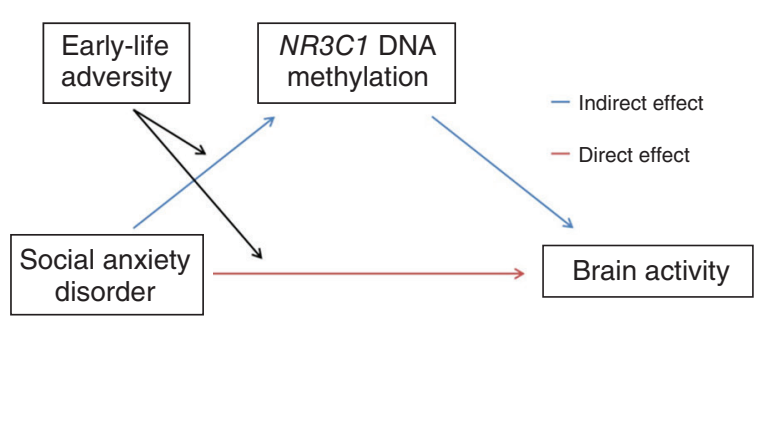

Figure 3: NR3C1 DNA methylation, neural correlates of the contrast "social anxiety disorder-related words versus neutral words" and their relationship in a moderated mediation model. (A) NR3C1 methylation for each group at site cg25708981. (B) Clusters of differential activation at an uncorrected threshold of $p<0.001$ and filtered for clusters of 10 or more voxels rendered on a standard brain. (C) Mean contrast estimates for each group extracted from the significant cluster. (D) Visualization of the direct and indirect effects of social anxiety disorder on brain activity mediated by NR3C1 DNA methylation and moderated by early-life adversity. Error bars depict standard errors of the mean; ${ }^{*} p<0.05$. au $=$ arbitrary units; $\mathrm{Ce}=$ control participants with low levels of early-life adversity; $\mathrm{CE}=$ control participants with high levels of early-life adversity; $\mathrm{Se}=$ participants with social anxiety disorder and low levels of early-life adversity; SE = participants with social anxiety disorder and high levels of early-life adversity. 
Table 4: Mediation of the effect of social anxiety disorder on brain activity by NR3C1 DNA methylation moderated by early-life adversity

\begin{tabular}{|c|c|c|c|c|}
\hline Effect & $\mathrm{B} \pm \mathrm{SEM}$ & $t$ & $p$ & $95 \% \mathrm{Cl}$ \\
\hline Social anxiety disorder $\rightarrow$ DNA methylation & $-0.008 \pm 0.004$ & -1.75 & 0.08 & -0.016 to 0.001 \\
\hline Early-life adversity $\rightarrow$ DNA methylation & $-0.008 \pm 0.004$ & -1.77 & 0.08 & -0.017 to 0.001 \\
\hline Social anxiety disorder $x$ early-life adversity $\rightarrow$ DNA methylation & $0.024 \pm 0.007$ & 3.63 & $<0.001$ & 0.011 to 0.037 \\
\hline DNA methylation $\rightarrow$ brain activity & $-13.377 \pm 6.497$ & -2.06 & 0.043 & -26.314 to -0.441 \\
\hline Social anxiety disorder $\rightarrow$ brain activity & $0.674 \pm 0.256$ & 2.63 & 0.010 & 0.164 to 1.184 \\
\hline Early-life adversity $\rightarrow$ brain activity & $0.958 \pm 0.270$ & 3.55 & $<0.001$ & 0.421 to 1.495 \\
\hline Social anxiety disorder $x$ early-life adversity $\rightarrow$ brain activity & $-1.506 \pm 0.403$ & -3.74 & $<0.001$ & -2.308 to -0.705 \\
\hline Social anxiety disorder $\times$ early-life adversity $\rightarrow$ DNA methylation $\rightarrow$ brain activity ${ }^{*}$ & $-0.315 \pm 0.164$ & - & - & -0.688 to $-0.050 \dagger$ \\
\hline $\begin{array}{l}\mathrm{Cl}=\text { confidence interval; } \mathrm{SEM}=\text { standard error of the mean. } \\
{ }^{*} \text { Indirect effect. } \\
\text { †Bootstrapped. }\end{array}$ & & & & \\
\hline
\end{tabular}

frontal gyrus $\left(x, y, z=-401034 ; F_{1,78}=21.05\right.$; cluster size 91 voxels, $728 \mathrm{~mm}^{3}$; $p_{\mathrm{FWE}}$ (whole brain) $=0.045$; Figure 3B). Seventy voxels from this cluster were located in the predefined region of interest $\left(p_{\mathrm{FWE}}=0.005\right)$, and 62 of these were within the cluster reported previously for the whole cohort $\left(p_{\mathrm{FWE}}<0.001\right)$. Decomposition of this interaction effect revealed an equivalent pattern of activation, as in the cluster for the whole cohort (Figure 3C).

A model of moderated mediation fit the data well $\left(F_{6,77}=\right.$ 4.92, $p<0.001$; Figure 3D). The analysis demonstrated that NR3C1 DNA methylation (cg25708981) partly mediated the relationship between social anxiety disorder and brain activity in the left middle frontal gyrus as a function of early-life adversity (Table 4).

\section{Discussion}

Investigating the association of social anxiety disorder and early-life adversity with hemodynamic activation in the neural system of attentional control, the key finding of the present study was the identification of 2 brain regions in the left and medial prefrontal cortex that exhibited an interaction between these factors during the processing of disorderrelevant stimuli. Concurrent DNA methylation analysis revealed that social anxiety disorder-related activity differences in the left middle frontal gyrus were partly mediated by NR3C1 DNA methylation as a function of early-life adversity. Importantly, these results provide the first evidence that there might be fundamental differences in the neurobiological processes that underlie social anxiety disorder, depending on the experience of early-life adversity.

\section{Neural interactions of social anxiety disorder and early-life adversity}

Although many studies have investigated the neural correlates of social anxiety disorder, findings have been partly contradictory, particularly in prefrontal brain regions. ${ }^{78}$ Although several studies have reported increased activation in the dorsolateral prefrontal cortex in association with social anxiety disorder in response to anxiety-related stimuli, ${ }^{39-41}$ others have observed decreased activity in the dorsolateral prefrontal cortex..$^{2,42}$ In the present study, we found that brain activity patterns associated with social anxiety disorder interacted with experiences of early-life adversity. Participants with social anxiety disorder and low levels of early-life adversity showed increased activation to disorder-relevant stimuli compared to healthy controls with equally low levels of early-life adversity; this increased activation did not occur in participants with social anxiety disorder and high levels of early-life adversity. Thus, our findings may suggest contingent differences in early-life adversity as one factor that contributes to the partly contradictory results of previous studies on the neural correlates of social anxiety disorder. Because the prevalence of early-life adversity is associated with economic factors and varies between countries, ${ }^{43}$ levels of earlylife adversity might differ between previous studies depending on their geographic and social settings. Moreover, our findings emphasize the importance of assessing early-life adversity, because it may constitute a relevant moderator of the neural underpinnings of social anxiety disorder. In line with this, previous research has associated early-life adversity with changes in brain activity and suggested early-life adversity as an uncontrolled confound in many neuroimaging studies on psychiatric disorders. ${ }^{14}$ It is not only that neural alterations might be attributed to social anxiety disorder when they might be more directly related to early-life adversity; it is also that the effects of social anxiety disorder and early-life adversity might interact (as observed in the present study), potentially leading to inconsistent or falsely negative results. Then again, only few studies that investigated brain activity differences in association with early-life adversity have controlled for psychiatric comorbidity. ${ }^{13}$ To our knowledge, the present study was the first neuroimaging analysis to investigate how neural activations associated with social anxiety disorder might interact with early-life adversity.

Both of the regions we identified coincided with parts of the neural network that is assumed to underlie supervisory attentional control, ${ }^{9}$ suggesting that the observed alterations were related to this psychological function, which has been causally associated with the maintenance of social anxiety on the basis of empirical data ${ }^{44}$ and psychological models. ${ }^{45,46}$ In contrast, no brain regions outside of the attentional control network exhibited any interaction effect. 
Our data suggest that participants with social anxiety disorder and low levels of early-life adversity and controls with high levels of early-life adversity engaged these brain regions more during attentional control in the context of negative emotional processing, and most prominently in the context of social anxiety disorder-related information processing. This finding might be indicative of a compensatory mechanism, with increased attentional effort as a consequence of increased automatic attention capture through this type of emotional information. However, we did not observe such increased neural responsiveness in participants with social anxiety disorder and high levels of earlylife adversity, whose activity patterns resembled control participants with low levels of early-life adversity in the identified regions. This initially counterintuitive reversal of increased activity in participants with social anxiety disorder and early-life adversity was not specific to social anxiety disorder-related stimuli; it was also present for fear-related and generally negative stimuli. This finding might indicate a more general alteration in neural responsivity to negative emotional information compared to neutral information in participants with social anxiety disorder and high levels of early-life adversity.

A recent study on the interrelations of social anxiety, dissociative symptoms and early-life adversity might serve as a starting point for the interpretation of this unexpected finding. It demonstrated that early-life adversity moderated the association between social anxiety and dissociative symptoms: ${ }^{47}$ a positive correlation was observed in participants with high levels of early-life adversity, but not in participants with low levels of early-life adversity. Dissociation, which describes the feeling of emotional or physical detachment, is regarded as a defence mechanism to avoid unpleasant emotional states, typically occurring after the experience of childhood trauma..$^{48}$ From the perspective of this line of research, the observed pattern with a generally reduced neural responsiveness to negative emotional stimuli relative to neutral stimuli might be related to a higher degree of dissociation in participants with social anxiety disorder and high levels of early-life adversity.

Recent research on the neural correlates of early-life adversity revealed effects that were selective for different subtypes of early-life adversity: emotional but not physical maltreatment has been associated with increased activity in the ventrolateral and dorsolateral prefrontal cortex. ${ }^{49}$ Concordantly, we observed significant positive correlations between brain activity and early-life adversity in participants without social anxiety disorder, for the emotional aspects of early-life adversity, but not the physical aspects. Interestingly, the negative correlation between brain activity and early-life adversity in participants with social anxiety disorder was also correlated with an emotional facet of early-life adversity (i.e., emotional neglect), but none of the physical subtypes of early-life adversity. This finding was also in line with previous research, indicating a more prominent contribution of emotional trauma to the psychopathology of social anxiety disorder compared to physical or sexual maltreatment. ${ }^{50}$ Therefore, the interaction ob- served in the present study might be more strongly related to emotional rather than physical or sexual aspects of childhood trauma.

In contrast to the interaction between social anxiety disorder and early-life adversity at the neural level, participants' response behaviour in the emotional Stroop task evidenced a main effect of social anxiety disorder driven by faster reaction times in participants with social anxiety disorder. It is conceivable that this behavioural effect was based on increased motivation to perform well in the experimental task, elicited by a central feature of social anxiety disorder pathology - namely feared scrutiny by others, such as the experimenters outside the scanner room.

\section{Epigenetic mediation of the interaction between social anxiety disorder and early-life adversity}

It has been suggested that epigenetic modifications of the gene encoding the glucocorticoid receptor, $N R 3 C 1$, physiologically mediate the long-term effects of early-life adversity on the functioning of the hypothalamic-pituitary-adrenal axis. ${ }^{51}$ We observed that DNA methylation at $1 \mathrm{CpG}$ site in NR3C1 mediated differential activity in the left middle frontal gyrus associated with social anxiety disorder as a function of early-life adversity. Participants with social anxiety disorder and low levels of early-life adversity showed decreased NR3C1 DNA methylation compared to control participants with equally low levels of early-life adversity. In contrast, in participants with high levels of early-life adversity, NR3C1 DNA methylation patterns were reversed. If one cautiously assumed that lower DNA methylation levels were associated with increased gene expression of $N R 3 C 1$, the corresponding increase in glucocorticoid receptors might be correlated with increased sensitivity to glucocorticoids. Binding of released glucocorticoids to the glucocorticoid receptor allows for regulation of the expression of glucocorticoid-responsive genes $^{52}$ and plays a critical role in the negative feedback of the hypothalamic-pituitary-adrenal axis. ${ }^{53}$ The CpG site reported here - cg25708981, within intron 2 of the NR3C1 gene - has previously been found to be hypomethylated in buccal cells in response to exposure to a synthetic glucocorticoid. ${ }^{54}$ However, previous studies have shown a reduced activity in the hypothalamic-pituitary-adrenal axis in response to social stress in individuals with a history of early-life adversity. ${ }^{55}$ In this regard, increased NR3C1 DNA methylation in those with social anxiety disorder and a history of earlylife adversity might represent an adaptation to repeated and enduring exposure to increased cortisol levels and be part of the underlying molecular basis for the reduced reactivity of the left middle frontal gyrus to negative emotional stimuli.

\section{Limitations}

The novel findings of our work provide a foundation for several strains of research to validate and extend our findings.

The absence of social anxiety disorder-associated behavioural correlates of altered attentional control in the present study somewhat limited the functional interpretation of our 
findings. The identified brain regions fit well with our a priori hypothesis of activity differences in the attentional control system, although we cannot rule out the possibility that other regions outside this network were involved. In future studies, designs with greater power to elicit behavioural correlates of altered attentional control in those with social anxiety disorder and early-life adversity (e.g., using multimodal stimuli more closely approximating real-life social threat $^{56-58}$ ) are needed to validate our interpretations and clarify the further functional implications of the observed effects. Beyond behavioural correlates, longitudinal studies that include interventions to modulate attentional alterations (e.g., attention bias modification training ${ }^{59}$ or noninvasive brain stimulation ${ }^{60}$ ) may help to explore the functional and clinical relevance of our results. Moreover, in the present study the variance for physical and sexual adversity was low, limiting the generalizability of our results to different types of early-life adversity.

The potential link of diminished emotional reactivity and dissociative symptoms in those with social anxiety disorder and high levels of early-life adversity represents another starting point for further research. This link appears to be very relevant, because symptoms of dissociation are present in many psychiatric disorders and have been connected to higher burden of illness and poorer treatment response. ${ }^{61}$

At the epigenetic level, we measured DNA methylation in whole blood and not in brain tissue, where it can be assumed to be more directly linked to brain activity. Nevertheless, the association of early-life adversity with differential NR3C1 DNA methylation has been demonstrated not only in peripheral blood ${ }^{51}$ but also in buccal cells ${ }^{62}$ and the hippocampus. ${ }^{63}$ Furthermore, if changes in NR3C1 DNA methylation are provoked by aberrant cortisol levels, ${ }^{54}$ the effects would not be limited to blood cells. Because cortisol passes the blood-brain barrier, it can be expected to have a similar effect on brain tissue, where it would be more directly related to the experience of stress and anxiety. Nevertheless, the observed differences in NR3C1 DNA methylation were relatively modest and the functional relevance of the identified CPG site needs to be clarified in studies with gene expression analyses. In addition, inclusion of further explanatory variables such as genotype data and subsequent trauma load might further improve the interpretability of the present findings. Moreover, because stress exposure during different time windows in childhood and adolescence may lead to different outcomes, ${ }^{64}$ future studies should assess the exact timing of early-life adversity to test whether it alters the moderating effect of earlylife adversity on NR3C1 DNA methylation and the brain activity patterns associated with social anxiety disorder. Similarly, the question arises whether adverse events later in life might also interact with the observed effects.

Investigating different aspects of the neurobiological mechanisms that underlie social anxiety disorder, we performed various statistical analyses in this study. Although we ensured multiple testing correction within separate analyses, such a comprehensive approach is generally accompanied with an increased risk of type I errors. The key findings of the present study need to be replicated in independent cohorts.

\section{Conclusion}

The present study provides the first evidence for essential differences in the neurobiological processes associated with social anxiety disorder, depending on a history of early-life adversity. Our data suggest that long-lasting effects of early-life adversity might be encoded in NR3C1 DNA methylation patterns and manifest in altered brain activity in the attentional control network during confrontation with anxiety-related stimuli.

Acknowledgements: The authors thank Danuta Altpaß, Gisbert Farger and Katia E. Ramadori for technical assistance. They also thank Cieslik and colleagues for sharing the results of their meta-analysis. They acknowledge support from Deutsche Forschungsgemeinschaft (DFG, NI 1332/6-1, FA 361/25-1, KR 4398/5-1) and the Open Access Publishing Fund of the University of Tübingen. The authors also wish to express their appreciation to all participants.

Affiliations: From the Department of Psychiatry and Psychotherapy, Tübingen Center for Mental Health, Eberhard Karls University of Tübingen, Tübingen, Germany (Wiegand, Munk, Drohm, Fallgatter, Nieratschker, Kreifelts); the International Max Planck Research School for Cognitive and Systems Neuroscience, University of Tübingen, Tübingen, Germany (Wiegand); the Department of Biology, Technische Universität Darmstadt, Darmstadt, Germany (Munk); the Department of Medical Genetics, University of British Columbia-BC Children's Hospital Research Institute, Vancouver, BC (MacIsaac, Kobor); and the Werner Reichardt Centre for Integrative Neuroscience, Tübingen, Germany (Nieratschker).

Competing interests: None declared.

Contributors: A. Fallgatter, V. Nieratschker and B. Kreifelts conceived and designed the study. A. Wiegand, M. Munk and S. Drohm recruited the participants and contributed to data acquisition. J. MacIsaac and M. Kobor contributed to the generation of the DNA methylation data. A. Wiegand and B. Kreifelts analyzed the data. A. Wiegand wrote the manuscript. M. Munk, S. Drohm, A. Falgatter, J. MacIsaac, M. Kobor, V. Nieraschker and B. Kreifelts revised the manuscript substantially. All authors approved the final version to be published and can certify that no other individuals not listed as authors have made substantial contributions to the paper.

Content licence: This is an Open Access article distributed in accordance with the terms of the Creative Commons Attribution (CC BY-NC-ND 4.0) licence, which permits use, distribution and reproduction in any medium, provided that the original publication is properly cited, the use is noncommercial (i.e., research or educational use), and no modifications or adaptations are made. See: https://creativecommons.org/licenses/by-nc-nd/4.0/

\section{References}

1. Heinrichs N, Hofmann SG. Information processing in social phobia: a critical review. Clin Psychol Rev 2001;21:751-70.

2. Evans KC, Wright CI, Wedig MM, et al. A functional MRI study of amygdala responses to angry schematic faces in social anxiety disorder. Depress Anxiety 2008;25:496-505.

3. Blair KS, Geraci M, Otero M, et al. Atypical modulation of medial prefrontal cortex to self-referential comments in generalized social phobia. Psychiatry Res 2011;193:38-45.

4. Schmidt S, Mohr A, Miltner WH, et al. Task-dependent neural correlates of the processing of verbal threat-related stimuli in social phobia. Biol Psychol 2010;84:304-12.

5. Boehme S, Ritter V, Tefikow S, et al. Neural correlates of emotional interference in social anxiety disorder. PLoS One 2015;10:e128608. 
6. Etkin A, Wager TD. Functional neuroimaging of anxiety: a metaanalysis of emotional processing in PTSD, social anxiety disorder, and specific phobia. Am J Psychiatry 2007;164:1476-88.

7. Bruehl AB, Delsignore A, Komossa K, et al. Neuroimaging in social anxiety disorder - a meta-analytic review resulting in a new neurofunctional model. Neurosci Biobehav Rev 2014;47:260-80

8. Freitas-Ferrari MC, Hallak JE, Trzesniak C, et al. Neuroimaging in social anxiety disorder: a systematic review of the literature. Prog Neuropsychopharmacol Biol Psychiatry 2010;34:565-80.

9. Cieslik EC, Mueller VI, Eickhoff CR, et al. Three key regions for supervisory attentional control: evidence from neuroimaging meta-analyses. Neurosci Biobehav Rev 2015;48:22-34.

10. Kessler RC, McLaughlin KA, Green JG, et al. Childhood adversities and adult psychopathology in the WHO World Mental Health Surveys. Br J Psychiatry 2010;197:378-85.

11. Günther V, Dannlowski U, Kersting A, et al. Associations between childhood maltreatment and emotion processing biases in major depression: results from a dot-probe task. BMC Psychiatry 2015;15:1-9.

12. Hart H, Lim L, Mehta M, et al. Altered fear processing in adolescents with a history of severe childhood maltreatment: an fMRI study. Psychol Med 2018;48:1092-101.

13. Hart H, Rubia K. Neuroimaging of child abuse: a critical review. Front Hum Neurosci 2012;6:52.

14. Teicher MH, Samson JA. Annual research review: enduring neurobiological effects of childhood abuse and neglect. J Child Psychol Psychiatry 2016;57:241-66.

15. Heim C, Binder EB. Current research trends in early life stress and depression: review of human studies on sensitive periods, geneenvironment interactions, and epigenetics. Exp Neurol 2012; 233:102-11.

16. Jaenisch R, Bird A. Epigenetic regulation of gene expression: how the genome integrates intrinsic and environmental signals. Nat Genet 2003;33 Suppl:245-54.

17. Turecki G, Meaney MJ. Effects of the social environment and stress on glucocorticoid receptor gene methylation: a systematic review. Biol Psychiatry 2016;79:87-96.

18. Tyrka AR, Parade S, Welch E, et al. Methylation of the leukocyte glucocorticoid receptor gene promoter in adults: associations with early adversity and depressive, anxiety and substance-use disorders. Transl Psychiatry 2016;6:e848-848

19. Tsigos C, Chrousos GP. Hypothalamic-pituitary-adrenal axis, neuroendocrine factors and stress. J Psychosom Res 2002;53:865-71.

20. Wittchen H-U, Zaudig M, Fydrich T. Strukturiertes klinisches Interview für DSM-IV. Achse I und II. Handanweisung. Hogrefe: Göttingen; 1997.

21. Stangier U, Heidenreich T. Liebowitz social anxiety scale. Göttingen, Germany: Hogrefe; 2005.

22. von Glischinski M, Willutzki U, Stangier U, et al. Liebowitz Social Anxiety Scale (LSAS): optimal cut points for remission and response in a German sample. Clin Psychol Psychother 2018;25:465-73.

23. Wingenfeld K, Spitzer C, Mensebach $C$, et al. The German version of the Childhood Trauma Questionnaire (CTQ): preliminary psychometric properties. Psychother Psychosom Med Psychol 2010;60:442-50.

24. Bernstein DP, Fink L. Childhood trauma questionnaire: a retrospective self-report. Manual. New York: Harcourt Brace \& Company; 1998.

25. Laux L. Das State-Trait-Angstinventar (STAI): theoretische grundlagen und handanweisung. Beltz: Weinheim; 1981.

26. Hautzinger M, Keller F, Kühner C, Beck AT. Beck DepressionsInventar: BDI II. Manual. Frankfurt am Main: Pearson Assessment; 2009.

27. Team RCR. A language and environment for statistical computing. Vienna, Austria: R Foundation for Statistical Computing; 2018
28. Collins DL, Neelin P, Peters TM, et al. Automatic 3D intersubject registration of MR volumetric data in standardized Talairach space. J Comput Assist Tomogr 1994;18:192-205.

29. Friston KJ, Worsley KJ, Frackowiak RS, et al. Assessing the significance of focal activations using their spatial extent. Hum Brain Mapp 1994;1:210-20.

30. Slotnick SD. Cluster success: fMRI inferences for spatial extent have acceptable false-positive rates. Cogn Neurosci 2017;8:150-5.

31. Wiegand A, Kreifelts B, Munk MH, et al. DNA methylation differences associated with social anxiety disorder and early life adversity. Transl Psychiatry 2021;11:1-10.

32. Triche TJ Jr, Weisenberger DJ, Van Den Berg D, et al. Low-level processing of Illumina Infinium DNA methylation beadarrays. Nucleic Acids Res 2013;41:e90.

33. Teschendorff AE, Marabita F, Lechner M, et al. A beta-mixture quantile normalization method for correcting probe design bias in Illumina Infinium $450 \mathrm{k}$ DNA methylation data. Bioinformatics 2013;29:189-96.

34. Johnson WE, Li C, Rabinovic A. Adjusting batch effects in microarray expression data using empirical Bayes methods. Biostatistics 2007;8:118-27.

35. Jones MJ, Islam SA, Edgar RD, et al. Adjusting for cell type composition in DNA methylation data using a regression-based approach. Methods Mol Biol 2017;1589:99-106.

36. Houseman EA, Accomando WP, Koestler DC, et al. DNA methylation arrays as surrogate measures of cell mixture distribution. BMC Bioinformatics 2012;13:86.

37. Hayes AF. Introduction to mediation, moderation, and conditional process analysis: a regression-based approach. New York: Guilford Publications; 2017.

38. Braun PR, Han S, Hing B, et al. Genome-wide DNA methylation comparison between live human brain and peripheral tissues within individuals. Transl Psychiatry 2019;9:47.

39. Blair K, Shaywitz J, Smith BW, et al. Response to emotional expressions in generalized social phobia and generalized anxiety disorder: evidence for separate disorders. Am J Psychiatry 2008;165:1193-202.

40. Blair K, Geraci M, Devido J, et al. Neural response to self- and other referential praise and criticism in generalized social phobia. Arch Gen Psychiatry 2008;65:1176-84.

41. Yoon KL, Fitzgerald DA, Angstadt M, et al. Amygdala reactivity to emotional faces at high and low intensity in generalized social phobia: a 4-Tesla functional MRI study. Psychiatry Res 2007;154:93-8

42. Gentili C, Gobbini MI, Ricciardi E, et al. Differential modulation of neural activity throughout the distributed neural system for face perception in patients with Social Phobia and healthy subjects. Brain Res Bull 2008;77:286-92.

43. Viola TW, Salum GA, Kluwe-Schiavon B, et al. The influence of geographical and economic factors in estimates of childhood abuse and neglect using the Childhood Trauma Questionnaire: a worldwide meta-regression analysis. Child Abuse Negl 2016;51:1-11.

44. Amir N, Beard C, Taylor CT, et al. Attention training in individuals with generalized social phobia: a randomized controlled trial. J Consult Clin Psychol 2009;77:961.

45. Clark D, Wells A. A cognitive model of social phobia. In: Heimberg RG, Liebowitz MR, Hope DA, Schneier FR, editors. Social phobia: diagnosis, assessment, and treatment. New York: Guilford Press; 1995: 69-93.

46. Rapee RM, Heimberg RG. A cognitive-behavioral model of anxiety in social phobia. Behav Res Ther 1997;35:741-56.

47. Myers NS, Llera SJ. The role of childhood maltreatment in the relationship between social anxiety and dissociation: a novel link. J Trauma Dissociation 2020;21:319-36.

48. Thomas PM. Protection, dissociation, and internal roles: modeling and treating the effects of child abuse. Rev Gen Psychol 2003;7:364-80. 
49. Cassiers LL, Sabbe BG, Schmaal L, et al. Structural and functional brain abnormalities associated with exposure to different childhood trauma subtypes: a systematic review of neuroimaging findings. Front Psychiatry 2018;9:329.

50. Kuo JR, Goldin PR, Werner K, et al. Childhood trauma and current psychological functioning in adults with social anxiety disorder. J Anxiety Disord 2011;25:467-73

51. Perroud N, Paoloni-Giacobino A, Prada P, et al. Increased methylation of glucocorticoid receptor gene (NR3C1) in adults with a history of childhood maltreatment: a link with the severity and type of trauma. Transl Psychiatry 2011;1:e59.

52. Oakley RH, Cidlowski JA. The biology of the glucocorticoid receptor: new signaling mechanisms in health and disease. J Allergy Clin Immunol 2013;132:1033-44.

53. Gjerstad JK, Lightman SL, Spiga F. Role of glucocorticoid negative feedback in the regulation of HPA axis pulsatility. Stress 2018; 21:403-16.

54. Braun PR, Tanaka-Sahker M, Chan AC, et al. Genome-wide DNA methylation investigation of glucocorticoid exposure within buccal samples. Psychiatry Clin Neurosci 2019;73:323-30.

55. Bunea IM, Szentágotai-Tatar A, Miu AC. Early-life adversity and cortisol response to social stress: a meta-analysis. Transl Psychiatry 2017;7:1274.

56. Kreifelts B, Brück C, Ritter J, et al. They are laughing at me: cerebral mediation of cognitive biases in social anxiety. PLoS One 2014;9:e99815
57. Kreifelts B, Weigel L, Ethofer T, et al. Cerebral resting state markers of biased perception in social anxiety. Brain Struct Funct 2019;224:759-77.

58. Carlson JM, Conger S, Sterr J. Auditory distress signals potentiate attentional bias to fearful faces: evidence for multimodal facilitation of spatial attention by emotion. J Nonverbal Behav 2018;42:417-26.

59. Heeren A, Mogoase C, Philippot P, et al. Attention bias modification for social anxiety: a systematic review and meta-analysis. Clin Psychol Rev 2015;40:76-90.

60. Heeren A, Billieux J, Philippot P, et al. Impact of transcranial direct current stimulation on attentional bias for threat: a proofof-concept study among individuals with social anxiety disorder. Soc Cogn Affect Neurosci 2017;12:251-60.

61. Lyssenko L, Schmahl C, Bockhacker L, et al. Dissociation in psychiatric disorders: a meta-analysis of studies using the dissociative experiences scale. Am J Psychiatry 2018;175:37-46.

62. Braithwaite EC, Kundakovic M, Ramchandani P, et al. Maternal prenatal depressive symptoms predict infant NR3C1 $1 \mathrm{~F}$ and BDNF IV DNA methylation. Epigenetics 2015;10:408-17.

63. McGowan PO, Sasaki A, D'alessio AC, et al. Epigenetic regulation of the glucocorticoid receptor in human brain associates with childhood abuse. Nat Neurosci 2009;12:342.

64. Lupien SJ, McEwen BS, Gunnar MR, et al. Effects of stress throughout the lifespan on the brain, behaviour and cognition. Nat Rev Neurosci 2009;10:434-45. 